\title{
EDUCAÇÃO PARA A SAUDE A GRUPO DE OBESOS
}

\author{
Glaci de Oliveira Pinto Vargas* \\ Suzana Fiore Scain **
}

$\operatorname{ReBEn} / 06$

VARGAS, G.O.P. e Colaboradora - Educação para a Saúde a Grupo de Obesos. Rev. Bras. Enf.: RS, 36: $38-49,1983$.

\section{RESUMO}

Este trabalho foi realizado por enfermeiras que atuam a nível ambulatorial, num Programa de Adulto, no Serviço de Saúde Pública, de um hospital geral de Porto Alegre, RS. É uma contribuição original de uma pesquisa descritivo analítica, com grupos de clientes obesos, atendidos através de cursos de educação para a saúde. Pretende-se avaliar quatro aspectos sem ordem de prioridade: a) interesse da clientela pela educação da saúde, em grupo; b) impressão, em relação aso conteúdos desenvolvidos; c) redução do peso; d) mudança de algum hábito de vida.

\section{1 - INFORMAÇÃO}

O atendimento ambulatorial, realizado por enfermeiros do Programa de Enfermagem na Sáde do Adulto, do Serviço de Saúde Pública, de um hospital geral, com grupo de clientes obesos, desenvolve ações educativas estimulando o autocuidado a domicílio. Este atendimento visa essencialmente a redução de danos controláveis e complicações que af etam os obesos.

O Serviço de Enfermagem de Saúde Pública visa recrutar a comunidade para desenvolver hábitos sadios de vida em relação à obesidade, of erecendo cursos de educação para a saúde. O curso dá informações a este grupo, considerando a dificuldade do cliente em diferenciar informação adequada e promoção de anúncios e conceitos populares, proporcionando aos obesos maior segurança na escolha do seu tratamento e aceitação da obesidade.

Pretende-se, baseados na literatura específica e experiência profissional, avaliar quatro aspectos que não obedecem ordem de prioridade: interesse da clientela pela educação para a saúde em grupo (através da procura do cliente ao serviço); impressão do cliente em relação aos conteúdos desenvolvidos; redução de peso e mudança de algum hábito de vida.

A educação para a saúde, feita através de cursos para clientes obesos, apresenta os conteúdos de modo gradual e contínuo, em situações de ensino-aprendizagem. A avaliação dos conteúdos fazse por instrumento pré-elaborados.

* Enfermeira do Serviço de Enfermagem de Saúde Pública do Hospital de Clínicas de Porto Alegre, especializada em Administração Hospitalar e Enfermagem do Trabalho.

** Enfermeira do Serviço de Enfermagem de Saúde Pública do Hospital de Clínicas de Porto Alegre, especializada em Enfermagem de Saúde do Adulto e Metodologia do Ensino Superior. 
VARGAS, G.O.P. e Colaboradora - Educação para a Saúde a Grupo de Obesos. Rev. Bras. Enf.: RS, 36: $38-49,1983$.

\section{2 - REVISÃO DA LITERATURA}

Segundo a XII Assembléia Mundial de Saúde, educação para a saúde é:

"A soma de todas aquelas experiências de um indivíduo, que modificam sua atitude ou comportamento com respeito à saúde e os processos e esforços para reduzir essas modificações". (10).

BRAVO (1958) definiu ... "método no trabalho sanitário, com a intenção não de prestar serviços aos indivíduos, mas de ajudá-los a remover certos hábitos do seu comportamento e a adotar para si uma atitude sadia para com a vida".

SILVA (1968) "a educação é um processo bilateral, no qual atuam educador e educando e sua finalidade é de ajudar os indivíduos a alcançarem a saúde, mediante seu comportamento e esforço".

A educação para a saúde faz parte do contexto da Saúde Pública e é meta a ser conquistada. Objetiva essencialmente ... "criar consciência da necessidade da mudança social, econômica e cultural, para superar os problemas de saúde determinados pelo grau de desenvolvimento e seus condicionantes; sensibilizar as pessoas para que se adaptem a novas condições de vida que signifiquem outros valores; a destruir crenças no que se refere à saúde, incrementando a racionalidade nesse campo e favorecendo caminhos para o cuidado com a saúde individual e coletiva; despertar o desejo do progresso na saúde, mediante a colaboração individual e coletiva" (10).

A educação para a saúde, como parte do contexto geral, sof re influência que vairam desde a urbanização, mudanças nos padrões de vida, fatores sociais, culturais e econômicos entre outros.

KRAUSZ (1971) af irma que "a urbanização leva parte da população ao subemprego, dificuldade no acesso a benefícios urbanos como: educação, saúde, saneamento do meio". "Os níveis de saúde de qualquer aglomerado urbano estão em função dos padrões de comportamento dos seus habitantes. Qualquer intervenção que vise uma mudança só terá sucesso à medida que levar em consideração o componente humano do problema em causa". Segundo MORAES (1977) "os fatores sociais e econômicos influem decisivamente sobre a educação. A inter-relação entre saúde e educação tem alta importância no progresso e desenvolvimento dos povos".

A receptividade da comunidade em relação à educação para a saúde a grupos não está desvinculada à receptividade da comunidade em relação a outros recursos de saúde of erecidos. "A mera presença de recursos(6)...não assegurará melhores níveis de saúde, se a população que se pretende assistir não for preparada para u sá-los convenientemente"..."e isto pressupõe a difusão de informação e a mudança de comportamentos relacionados com os conceitos de saúde e doença". MORAES (1977) afirmou que "nas últimas décad as tem-se procurado interessar a comunidade a participar dos programas de saúde. Esta, quando dificiente, constitui importante barreira ao desenvolvimento ..." cabe à conscientização dos indivíduos e das comunidades para, numa ação conjunta, modificarem atitudes e costumes da vida diária ..."." "E preciso, então, que as pessoas além de informadas, compreendam o que cada pessoa, grupo e comunidade podem fazer para atingir o objetivo visado e, assim adquirir atitudes, experiências e hábitos, em suma, adquirir maneiras de comportamento que bem as conduzam a uma saúde melhor e a uma vida mais longa e mais produtiva" (2). "A proliferação de produtos alimentares "naturais" e de clubes de ginástica, as proibições de fumar nos locais públicos, como nos aviões ... as corridas matinais, desconhecidas há 10 anos ... demonstram claramente que o público não é insensível às medidas preconizadas" $\left({ }^{9}\right)$. O que falta é a ênfase à educação para a saúde, uma vez que o melhoramento das comunicações e os progressos tecnológicos podem modificar o conceito que a população tem sobre a saúde". (12).

Destacamos a importância d a participação do enfermeiro na educação para a saúde, tanto a nível individual com o grupal. SILVA (1968) reforça "que o enfermeiro é um prof issional perfeitamente capacitado para desempenhar as funções de educação sanitária junto a grupos e à comunidade, quer pela natureza de seu trabalho, quer pelo tipo de formação profissional que ele receba. Atualmente, está sendo feito um esforço profissional - "professores, sociólogos, médicos. . . farmacêuticos, enfermeiros" - $\left.{ }^{19}\right)$, no sentido de reconhcer o valor e a necessidade da educação para a saúde.

Acredita-se na ef etividade e na validade da educação para a saúde a grupos aqui tratados:o de obesos. Ressalta-se que o atendimento a este grupo maior motivação e conscientização do cliente em relação ao seu dano próprio. KRAUSZ (1971) refere-se ao fato de que a educação em saúde ajuda a pessoa, individualmente ou em grupos, a desenvolver seu desejo de saúde, a conscientizar o seu problema de saúde pessoal e social, a absorver o conhecimento específico e através do aconselhamento e encorajamento necessários, aprender, a forma de aplicar este conhecimento. 
VARGAS, G.O.P. e Colaboradora - Educação para a Saúde a Grupo de Obesos. Rev. Bras. Enf.:RS, 36: $38-49,1983$.

A educação para a saúde a este grupo é feita através de uma programação - "um programa de saúde se elabora com a finalidade de se mudar uma situação por outra que se considera melhor" (01). Abordaremos neste programa, entre outros conteúdos: a atividade física sistemática e a educação alimentar, na tentativa de modificar hábitos de vida que trazem prejuízos para a saúde. "Agora nós sabemos que os estilos de vida são a origem do mau estado de nossa saúde . . . o álcool, o fumo . . . a falta de exercício físico ... são as principais causa. Entretanto, elas ainda não são reconhecidas, nem aceitas e são até ignoradas pelo grande público" (09).

Baseadas nisto é que pesamos a necessidade e a validade de desenvolver atividades que levem a um maior número de pessoas os conhecimentos que auxiliam o cuidado no tratamento. $O$ autor (09) reforça dizendo que "a educação tornou-se necessária para vencer idéias falsas, denunciar os perigos do fumo ... e demonstrar as vantagens de uma alimentação equilibrada, de uma conduta correta nos exercícios físicos".

Outro aspecto de importância, abordado a grupo de obesos, é o apoio emocional, por serem notórias as dificuldades emocionais desta clientela que, repetidas vezes, obstaculiza a decisão da realização do tratamento. CARSON RYN, citado por BASTOS (1958) enumera as emoções como um dos princípios básicos da aprendizagem: "se é que se aspira a mudança de comportamento do indivíduo e que as boas relações humanas são de suma importância na aprendizagem do adulto".

Nesta at ividade grupal faz-se avaliação com medidas objetivas, subjetivas e inferenciais.

GOMES (1979) descreve que as medidas de avaliação quase sempre são medidas indiretas, do efeito de um programa sobre uma determinada pessoa, família, grupos de comunidade,. FREEMAN e HOME (1962) identificam três tịpos de avaliação em saúde pública: objetiva, subjetiva ou de julgamento e inferencial.

Espera-se que este trabalho contribua para firmar a conviç̧ão de que a educação para a saúde a grupo de obesos é exeqüível e necessária dentro de um serviço de saúde pública, além de incentivada e divulgada.

\section{3 - MATERIAL E MÉTODO}

O presente trabalho é um estudo retrospectivo analítico baseado em dados obtidos, através de fichas de identificação, de controle e de avaliação (contínua e final) de cursos de educação para a saúde a clientes obesos do Serviço de Enfermagem de Saúde Pública, do programa de Enfermagem na Saúde do Adulto de um hospital geral. Foram analisadas as anotações feitas por ocasião dos cursos realizados no período de maio de 1981 a maio de 1982, numa totalidade de quatro cursos.

\section{1 - População:}

A população alvo para a execução deste estudo foi baseada entre os cliente obesos que frequentaram tais cursos. O programa de Enfermagem na Saúde do Adulto realiza tal assistência desde 1979.

\section{2 - Amostragem:}

Foi estudada a totalidade das fichas dos clientes obesos desde o momento da inscrição até a conclusão dos cursos, no período de 11 de maio de 1981 a 17 de maio de 1982, num total de 136 clientes.

A totalidade da clientela foi utilizada para analisar o interesse pela educação para a saúde em grupo. Utilizou-se os clientes que concluíram, para analisar as suas impressões em relação ao conteúdo desenvolvido, à redução de peso e à mudança de algum hábito de vida.

\section{3 - Fonte de Dados:}

Os dados foram colhidos das fichas de identificação (Anexo I)., de controle (Anexo II) e de avaliação (Anexo III).

Estas tichas são de uso da enfermeira e dos clientes. A ficha de identificação do cliente é preenchida pela enfermeira no período de divulgação, durante a inscrição do cliente, em média 15 dias, antecedendo o início dos cursos.

A ficha de controle é preenchida com dados antropométricos (peso) sinais vitais (PA, FC)e sintomas pela enfermeira, no primeiro e último encontros.

A ficha de avaliação consta de dados objetivos, solicitando avaliação do cliente a cada conteúdo desenvolvido, através de conceitos e sugestões, no final de cada encontro, sendo que quatro per- 
VARGAS, G.O.P. e Colaboradora - Educação para a Saúde a Grupo de Obesos. Rev. Bras. Enf.:RS, 36: $38-49,1983$.

guntas preenchidas no último encontro especificam o aproveitamento individual, aspectos positivos e negativos do curso.

\section{4 - Procedimentos:}

A realização da pesquisa.

Para a coleta de dados, observam-se os seguintes passos:

a) utilização dos dados registrados na ficha de identificação para comparação da clientela inscrita, daquela que conclui o curso.

b) utilização dos dados da ficha de controle para análise da evolução individual da clientela relativa à perda e da ficha de avaliação (pergunta 10);

c) utilização da ficha de avaliação para análise da impressão do cliente (dados objetivos), em relação ao conteúdo desenvolvido e mudança dos seus hábitos de vida (dados objetivos inferenciais).

\section{5 - Processamentos de Dados:}

Para o processamento de dados apurou-se o número de clientes inscritos e dos que concluíram os cursos, através da ficha de inscrição, avaliação parcial e final.

Para fins de apuração dos dados da evolução em peso corporal, sentiu-se a necessidade de agrupá-los em intervalos de $4 \mathrm{~kg}$ em ordem decrescente, de $12 \mathrm{~kg}$ até aumento de peso.

Na Tabela 4 foram agrupados "outros hábitos de vida" para maior clareza da tabela.

0 termo obesidade ( $20 \%$ acima do peso normal) neste trabalho inclui o termo excesso de peso (até $20 \%$ do peso normal) embora sejam conceitos diferentes, para facilitar a leitura do texto e não torná-lo cansativo.

$\mathrm{Na}$ Tabela 5 foram agrupados os aspectos positivos, conforme descrição subjetiva da clientela, já que a característica da pergunta requer uma resposta aberta.

\section{4 - RESULTADOS E DISCUSSÃO}

Os resultados da pesquisa representam o produto da educação para a saúde a grupo de clientes obesos, através de cursos, a nível ambulatorial, dentro de um programa de Saúde do Adulto.

A Tabela 1 mostra a afluência da clientela para este tipo de assistência; a Tabela 2 a perda de peso corporal dos clientes que conchuiram os cursos; a Tabela 3 mostra a avaliação parcial dos conteúdos desenvolvidos realizada pelos próprios clientes, a Tabela 4 a avaliação final em relação a mudança nos hábitos de vida e a última, os aspectos positivos evidenciados pela clientela.

Na Tabela 4, a avaliação prenchida pela clientela possibilita a escotha de mais de um item, no entanto, o percentual caculado foi sobre 83 clientes concluentes.

Na mesma tabela, o item "outros" engloba a escolha feita pela clientela sem discriminar qual hábito foi modificado.

$\mathrm{Na}$ Tabela 5 não foram distribuídos os aspectos negativos, visto que somente três clientes $(3,6 \%)$ fizeram menção a tal - técnica de ensino ao desenvolver os conteúdos apresentados, legibilidade deficientes dos polígrafos of erecidos, horário do curso no sentido de serem of erecidos outros para maior acesso da comunidade, ventilação do ambiente e verbalização individual da clientela frente a seus problemas em relação à obesidade.

Comenta-se nesta mesma tabela o aspecto - "não respondido" - onde foram encontradas respostas como "todos os aspectos são positivos", "nenhum aspecto negativo", "o curso só apresentou aspectos positivos", etc., sem listá-los, além dos que deixaram de responder. 
VARGAS, G.O.P. e Colaboradora - Educação para a Saúde a Grupo de Obesos. Rev. Bras. Enf.:RS, 36: $38-49,1983$.

\section{TABELA I}

Número de clientes inscritos e concludentes nos cursos para obesos, promovidos pelo Serviço de Enfermagem de Saúde Pública, na área da Saúde do Adulto, num hospital geral, de 11 de maio de 1981 a 17 de maio de 1982, em Porto Alegre.

\begin{tabular}{c|c|c|c|c}
\hline & I N S C R I T O S & \multicolumn{2}{c}{ CONCLUENTES } \\
\hline & No & $\%$ & No & $\%$ \\
\hline I CURSO & 32 & 100,0 & 26 & 82,0 \\
\hline II CURSO & 22 & 100,0 & 12 & 54,5 \\
\hline III CURSO & 38 & 100,0 & 25 & 66,5 \\
\hline IV CURSO & 44 & 100,0 & 20 & 45,4 \\
\hline T O T A L & 136 & 100,0 & 83 & 61,0 \\
\hline
\end{tabular}

Observa-se na Tabela que $61 \%$ da clientela inscrita concluiu os cursos, sendo que $39 \%$ foi desistente, notando-se maior concentração no primeiro e terceiro cursos.

TABELA 2

Perda de peso corporal dos clientes ao final dos cursos para obesos, realizados pelo Serviço de Enfermagem de Saúde Pública, no programa da Saúde do Adulto, de um hospital geral,, de 11 de maio de 1981 a 17 de maio de 1982, em Porto Alegre.

\begin{tabular}{l|c|c}
\hline PERDA DE PESO & No DE CLIENTES & $\%$ \\
\hline $12 \longmapsto \quad 8$ & 1 & 8,3 \\
\hline $8 \longmapsto 4$ & 15 & 18,0 \\
\hline $4 \longmapsto \begin{array}{l}\text { manutenção } \\
\text { de peso }\end{array}$ & 54 & 65,0 \\
\hline Aumento de peso & 13 & 15,6 \\
\hline T O T A L & 83 & 100,0 \\
\hline
\end{tabular}

Os cursos de educação para a saúde, a obesos, possibilitaram a $65 \%$ dos clientes uma perda de peso corporal de até $4 \mathrm{~kg}$ ou sua manutenção e que $15 \%$ deles apresentaram aumento de peso. 
VARGAS, G.O.P. e Colaboradora - Educação para a Saúde a Grupo de Obesos. Rev. Bras. Enf.: RS, 36: $38-49,1983$.

\section{TABELA 3}

Avaliação parcial dos conteúdos desenvolvidos, realizada pelos clientes dos cursos para obesos, promovidos pelo Serviço de Enfermagem de Saúde Pública, no program da Saúde do Adulto, de um hospítal geral, de 11 de maio de 1981 a 17 de maio de 1982, em Porto Alegre.

\begin{tabular}{|c|c|c|c|c|c|c|c|c|}
\hline \multirow{2}{*}{$\begin{array}{l}\text { ENCONTROS } \\
\text { CONCEITUAÇÃO }\end{array}$} & \multicolumn{2}{|c|}{ OTIMO } & \multicolumn{2}{|c|}{ BOM } & \multicolumn{2}{|c|}{ REGULAR } & \multicolumn{2}{|c|}{$\begin{array}{l}\text { SEM AVALIAÇÃO POR } \\
\text { AUSÊNCIA CLIENTES }\end{array}$} \\
\hline & N? & $\%$ & N? & $\%$ & No & $\%$ & N? & $\%$ \\
\hline 19 Encontro & 33 & 39,7 & 25 & 30,1 & 3 & 3,6 & 22 & 26,5 \\
\hline 2\% Encontro & 65 & 78,3 & 9 & 10,8 & 1 & 1,2 & 8 & 9,6 \\
\hline 39 Encontro & 56 & 67,4 & 17 & 20,4 & - & - & 10 & 12,0 \\
\hline 4? Encontro & 62 & 74,6 & 10 & 12,0 & - & - & 11 & 13,2 \\
\hline 5 ․ Encontro & 55 & 66,2 & 13 & 15,6 & 1 & 1,2 & 14 & 16,8 \\
\hline 6 ? Encontro & 65 & 78,3 & 7 & 8,4 & 1 & 1,2 & 10 & 12,0 \\
\hline 79. Encontro & 51 & 61,4 & 14 & 16,8 & 1 & 1,2 & 8 & 9,6 \\
\hline
\end{tabular}

A avaliação feita pela clientela, em relação aos conteúdos desenvolvidos, mostra que a maioria deu maior número de pontos ao conceito ótimo, destacando-se o 2 \% e 6 \% encontros, com percentual de $78,3 \%$. O percentual do conceito bom no 1 ? encontro foi de $30,1 \%$ - o maior desta faixa, o mesmo ocorrendo com o conceito regular $3,6 \%$ no mesmo encontro. Destaca-se que $26,5 \%$ da clientela não avaliou o 1 \% encontro. 0 total da clientela considerada é de 83 .

\section{TABELA 4}

Avaliação final, na mudança de hábitos de vida, realizada pela clientela dos cursos para obesos, promovidos pelo Serviço de Enfermagem de Saúde Pública, do programa da Saúde do Adulto, de um hospital geral de Porto Alegre, de 11 de maio de 1981 a 17 de maio de 1982.

\begin{tabular}{l|c|c}
\hline \multicolumn{1}{c|}{ MUDANÇA NO HÅBITO DE VIDA } & No & $\%$ \\
\hline Atividade física & 33 & 39,7 \\
\hline Alimentção & 53 & 63,8 \\
\hline Maneira de encarar a obesidade & 37 & 44,5 \\
\hline Outros hábitos & 6 & 7,2 \\
\hline Não respondido & 16 & 19,2 \\
\hline
\end{tabular}

A clientela modificou seu hábito alimentar em $63,8 \%$, seguido de $44,5 \%$ na maneira de encarar a obesidade. 
VARGAS, G.O.P. e Colaboradora - Educação para a Saúde a Grupo de Obesos. Rev. Bras. Enf.: RS, 36: $38-49,1983$.

\section{TABELA 5}

Aspectos positivos evidenciados pela clientela nos cursos para obesos, promovidos pelo Serviço de Enfermagem de Saúde Pública, do programa de Saúde do Adulto, de um hospital geral, de 11 de maio de 1981 a 17 de maio de 1982, em Porto Alegre.

\begin{tabular}{l|c|c}
\hline \multirow{2}{*}{\multicolumn{1}{c|}{ A S P E C T O S }} & \multicolumn{2}{c}{ POSITIVO } \\
\cline { 2 - 3 } & No & 12,0 \\
\hline Educação em grupo & 10 & 27,7 \\
\hline Estímulo para realização do tratamento & 23 & 19,2 \\
\hline Conteúdos desenvolvidos & 16 & 8,4 \\
\hline Formação de um clube ou associação para obesos & 7 & 42,1 \\
\hline Não respondido & 35 & 4,8 \\
\hline Apoio emocional & 4 & 6,0 \\
\hline Comunicação e integração do coordenador & 5 & \\
\hline
\end{tabular}

Verifica-se que $27,7 \%$ da clientela destacou o estírnulo para realização do tratamento, seguido de $19,2 \%$ em relação aos conteúdos desenvolvidos. Observa-se que $42,1 \%$ dos obesos não responderam a avaliação.

\section{CONCLUSŌES}

A afluência da comunidade na educação para a saúde a grupos de obesos é signif icativa. $\mathrm{Na}$ inscrição de 4 cursos para obesos, somou-se 136 clientes considerando a prematuridade deste tipo de assistência em nosso meio. Embora haja desistência no decorrer dos cursos, a maioria dos obesos concluiu os cursos, num total de 83 clientes (61\%). Os motivos conhecidos de disistência ou diminuição da freqüência, são as estações do ano em que são realizados os cursos; no inverno há maior ocorrência deste fator. Exemplificando, o segundo curso foi realizado de agosto a setembro de 1981 e a defasagem da inscrição para o curso e dos concluentes foi de $45 \%$. Nos meses de dezembro, janeiro e fevereiro nem são realizados cursos, por serem meses de férias e festas, diminuindo o afluxo da população aos serviços de saúde em geral. Citamos, ainda, doenças e viagens.

O objetivo deste trabalho não é comentar a respeito do custo destes cursos. Contudo, observou-se que o maior contingente de desistência foi no 4 ? curso, o único não pago, por questões administrativas. Concluindo-se que a clientela não valorizou a oportunidade of erecida.

A perda de peso corporal não é o objetivo principal destes cursos de educação para a saúde em grupo, mas se observa que 70 clientes perderam peso e a concentração maior foi de $4 \mathrm{~kg}$ a manutenção de peso. Constata-se, ainda, que 13 clientes que aumentaram de peso não modificaram o seu estilo de vida:

A clientela de obesos, quando avaliou os cursos, demonstrou aproveitamento e aceitação dos conteúdos desenvolvidos na maioria dos encontros.

O 1 \% encontro foi que recebeu menor pontuação em relação aos demais, porque foi o único que não desenvolveu conteúdos. Nele foi apresentado o cronograma, os participantes, a avaliação e outras considerações gerais. Nos demais encontros o conceito ótimo foi o predominante.

Observa-se que no 3 ? curso, ao 1 \% encontro, houve 22 clientes que não avaliaram, isto porque se inscreveram a partir do $2 \%$ encontro.

A maioria absoluta da clientela modificou seu estilo de vida, primeiramente em relação a hábitos alimentares, segu idos da maneira diferente de encarar a obesidade e atividade física. Houve mudanças em outros hábitos de vida, mas não foram especificados. 
VARGAS, G.O.P. e Colaboradora - Educação para a Saúde a Grupo de Obesos. Rev. Bras. Enf.: RS, 36: $38-49,1983$.

Nos cursos de educação para a saúde, os clientes obesos destacaram, na grande maioria, aspectos positivos como: estímulo na realização do tratamento e conteúdos desenvolvidos. Embora a formação de uma associação para obesos, a fim de dar continuidade à assistência destes clientes não tenha sido de maior destaque, deixou claro a necessidade desta entidade.

Conclui-se que no item "não respondido", a clientela encontrou dificuldades em responder, por ser descritivo.

Alcançou-se, desta maneira, os objetivos estabelecidos para o presente trabalho, podendo os resultados obtidos servirem de subsídios para a programação de outros grupos educativos, que devem fazer parte da programação feita pela enfermeira na assistência da saúde do adulto.

\section{SUMMARY}

This study was prepared by nurses who perform at outpatient level, on an Adult Health Program, in Public Health, at a general hospital in Porto Alegre, RS. It is an original contribution of a descriptive-analytic research, with groups of overweight patients, cared for through health education courses. It is here intended the evaluation of four aspects, without any priority order:interest demonstrated by the clients towards group education, their reaction to the subjects developed, weight reduction and translated by the alteration of some life habit.

\section{REFERÊNCIAS BIBLIOGRẢFICAS}

1 - ALVIN, Emergarda de F. - Considerações sobre o trabalho educativo num sistema médico sanitário educativo. Revista Brasileira de Enfermagem. Rio de Janeiro, 8 (3): 206-14, set. 1955.

2 - BASTOS, Nilo Chaves de Britto e GROSSMAN, JEROME. - Programa de educação sanitária. Revista Paulista de Hospitais. São Paulo, 6 (10): 11-7, out. 1958.

3 - BRAVO, A. L. - Health Education in Industry, Int. J. Health Edu. 1 (1): 16-24, 1968.

4 - FREEMAN, Ruth B. e HOLMES, Edward M. - In: Preparacion del pueblo para la salud pública. Administracion de los Serviços de la Salud Publica. México. Interamericana, 1962.

5. - GOMES, Daysi. Avaliação em Saúde. Revista Enfermagem em Novas Dimensões, São Paulo, 5 (3): 149-52, mai/jun., 1979.

6. - KRAUSZ, Rosa R. - Os desafios da urbanização para a educação sanitária. Revista Saúde PúbliCi. São Paulo, 5 (2): 285-9, dez. 1971.

7 - MITCHELL, Helen S. e t alli. - Nutrição, Rio de Janeiro, Interamericana, 1978.

8 - MORAES, Leovegildo L. de - Saú de/educação:u m binômio indivisível. Jornal de Pediatria, Rio de Janeiro, 42 (6): 48-54, jun. 1977.

9 - O HOSPITAL pode encarregar-se da educação sanitária. Vida Hospitalar, São Paulo, 10 (4): 230$1,1976$.

10 - PIMONT, Rosa Pavone. A educação em saúde. Boletin de la Oficina Sanitaria Panamericana. 82 (1): 14-21, maio, 1977.

11 - SILVA, Maria Jacyra N. D. - Sugestões para maior integração da educação sanitária no curso de graduação de enfermagem. Revista da Escola de Enfermagem da USP, São Paulo, 2 (1): 39-60, mar. 1968.

12 - SOBREIRA, Nilze Rodrigues. Enfermagem Comunitária. Rio de Janeiro, Internamericana, 1981. 
VARGAS, G.O.P. e Colaboradora - Educação para a Saúde a Grupo de Obesos. Rev. Bras. Enf.: RS, 36: $38-49,1983$.

\section{BIBLIOGRAFIAS CONSULTADAS}

1 - ARCHER, Sarah Helen e FLESHMAN. Ruth. - Enfermaria de Slud Comunitária. Colômbia. Carjaval, 1977.

2 - BASTIAN, Ernestine M. - Planejamento e Organização de um Programa Didático. Aplicação a situações de ensino no campo da saúde. São Paulo, USP, 1976.

3 - CAMPOS, Juarez de Queiroz. A tuação dos hospitais na promoção da saúde. Revista Paulista de Hospitais. São Paulo, 22 (2): 74-7, fev. 1974.

4 - COSTA, Léa L. da. - Saúde aprendizagem. Jornal de Pediatria. Rio de Janeiro, 44 (4): 258-60, abr. 1978.

5 - DOWLING, M. A. C. et alli. A modernização do ensino. Saúde do Mundo. Genebra: 12-5, abr. 1977.

6 - DOWLING. M. A. C. et alli. - Para u ma major adaptacion de los programas de enseñanza. Cronica de la OMS. Genebra, 30 (11): 505-8, nov. 1976.

7 - EL PROGRAMA de educacion sanitaria de la OMS. Cronica de la OMS. Genebra, 28 (9): 44453, set. 1974.

8 - EZ BAN, A. - Revolução sanitária. Saúde do Mundo. Genebra: 14:9, abr. 1975.

9 - GREGOR, A. e DOWLING, M. A. C. - Ensinando e aprendendo. Saúde do Mundo. Genebra: 147,jan. 1978.

10 - GOTTSCHALL, Carlos Antônio M. - Idéia sobre educação e desenvolvimento. Revista da Associação Médica do Rio Grande do Sul. Porto Alegre. 15 (2): 141-7. jun. 1971.

11 - KAMIYANA, Yoriko et alli. Educação para a saúd e: experiência de integração hospital-escola de enfermagem. Revista Enfermagem em Novas Dimensões. São Paulo, 3 (5): 300-5, set. - out., 1977.

12 - KNOPLICH, José. - Viva bem com a coluna que você tem. São Paulo, Ibrosa, 1980.

13 - MƯXFELDT, Léa C. F. - Assistência de Enfermagem ao cliente em clínica de atendimento externo. Revista do Hospital de Clínica de Porto Alegre, 1 (2): 107-110, 1982.

14 - ORGANIZACION de los serviços de educacion sanitaria. Cronica de 1a OMS. Genebra, 30 (1): 36-8, enero 1976.

15 - SEMINARIO sobre sistemas nacionais de informacion sanitaria. Cronica de 1a OMS. Genebra, 33 (9): 382-86, sep. 1979.

16 - SIlVA NETTO, Joaquim A. da. - Educação da Saúde. Jornal de Pediatria. Rio de Janeiro.33 (11/12): 249-53, nov/dez. - 1968. 
VARGAS, G.O.P. e Colaboradora - Educação para a Saúde a Grupo de Obesos. Rev. Bras. Enf.: RS, 36: $38-49,1983$.

\section{ANEXO I}

\section{FICHA DE IDENTIFICAÇÃO:}

Nome:

Enderȩ̧o:

Sexo:

Idade:

Grau de Instrução:

Profissão:

Renda Mensal:

Estado Civil:

Tem Filhos:

Se positivo, quantos e que idade:

Encaminhado por: 
VARGAS, G.O.P. e Colaboradora - Educação para a Saúde a Grupo de Obesos. Rev. Bras. Enf.: RS, 36: 38-49, 1983.

\section{ANEXO II}

FICHA DE CONTROLE:

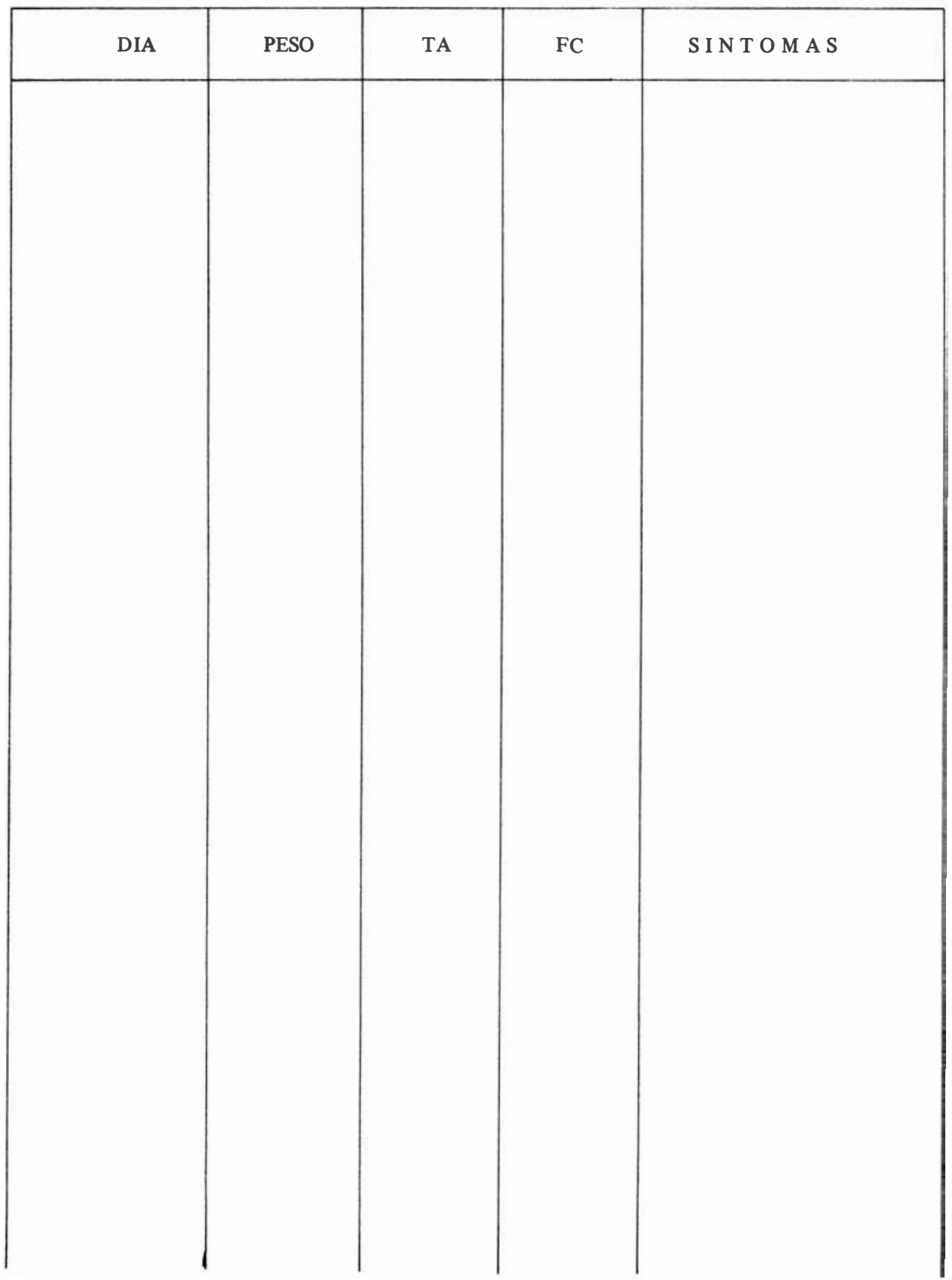


VARGAS, G.O.P. e Colaboradora - Educação para a Saúde a Grupo de Obesos. Rev. Bras. Enf.: RS, 36: $38-49,1983$.

\section{ANEXO III}

FICHA DE AVALIAÇĀO:

1 - Aula N +1 - Apresentação

Otimo $\square$ - Apresentação Bom

Regular $\square$

Insuficiente $\square$

2 - Aula No 2 - O que é obesidade, causas, conseqüências

\section{Otimo $\square$}

Bom $\square$

Regular $\square$

Insuficiente $\square$

Sugestões:

3 - Aula No 3 - Princípios básicos de nutrição

Otimo $\square \quad$ Bom $\square$

Regular $\square$

Insuficiente $\square$

Sugestões:

4 - Aula No. 4 - Dieta hipocalórica

Otimo $\square$ Bom

Sugestões:

Regular $\square$

Insuficiente $\square$

5 - Aula No 5 - Erros alimentares Otimo $\square$

Bom

Regular $\square$

Insuf iciente $\square$

Sugestões:

6 - Aula No 6 - Atividade física e recreação

Otimo $\square \quad$ Bom $\square$

Regular

Insuficiente

Sugestões:

7 - Aula No 7 - Manutenção de peso

Ótimo $\square \quad$ Bom $\square$

Sugestões

Regular

Insuficiente $\square$

8 - O que o curso significa para você?

9 - O curso mudou algum hábito da sua vida?

Qual? Atividade física

Alimentação

Outros

10 - Como foi sua evolução em relação ao peso?

Negativa $\square$

Positiva

Nenhuma

11 - Quais os aspectos positivos e negativos do curso. 